\title{
ESTRUCTURA Y COMPOSICIÓN DE UN ENSAMBLAJE DE AVES ASOCIADAS AL RÍO MAIMÓN, REPÚBLICA DOMINICANA
}

\author{
Structure and composition of bird assemblages associated \\ with the Maimón river, Dominican Republic
}

\begin{abstract}
Hodali Almonte-Espinosa
Museo Nacional de Historia Natural “Prof. Eugenio de Jesús Marcano”. Calle César Nicolás Penson, Plaza de la Cultura Juan Pablo Duarte, 10204, Santo Domingo, República Dominicana; (D orcid.org/0000-0002-4151-7175. h.almonte@mnhn.gov.do
\end{abstract}

[Recibido: 4 de agosto, 2021. Aprobado para publicación: 21 de octubre, 2021]

\section{RESUMEN}

Se analizó la composición y estructura de un ensamblaje de aves asociadas a cuatro localidades ubicadas en los márgenes del río Maimón: confluencia del río Maimón con río Yuna, carretera Maimón Piedra Blanca, Piedra Blanca y Los Plátanos. Se registró un total de 598 individuos pertenecientes a 51 especies y 28 familias de aves. La localidad de Maimón Piedra Blanca registró los valores más altos de diversidad usando el índice de Shannon $\left(\mathrm{H}^{\prime}=3.36\right)$, igualmente fue la estación con mayor abundancia y riqueza: 217 individuos y 43 especies respectivamente. Por el contrario, la estación ubicada en Los Plátanos presentó la menor diversidad de acuerdo con el índice de Shannon $\left(\mathrm{H}^{\prime}=2.92\right)$. Además, esta estación mostró la menor riqueza: 29 especies. Se registraron siete gremios y 15 categorías tróficas. Los carnívoros y omnívoros fueron los grupos más diversos y abundantes. Se registraron diferencias significativas en la abundancia relativa de aves por gremios tróficos y por área de estudio $\left(\mathrm{X}^{2}=43.32, \mathrm{df}=18, \mathrm{p}=\leq 0.01\right)$. No se encontró diferencia en la riqueza de especies por gremio trófico $\left(\mathrm{X}^{2}=6.67 \mathrm{df}=18, \mathrm{p}=0.99\right)$. El número de categorías tróficas fue similar en las zonas evaluadas $\left(\mathrm{X}^{2}=39.34, \mathrm{~d} f=42, \mathrm{p}=0.58\right)$. Se concluye que el ensamblaje de aves asociado al río Maimón se distribuye de forma equitativa. Además, tanto su composición como estructura están compuestas principalmente, por especies generalistas de hábitats y de amplia distribución. Finalmente, a pesar de que las áreas de estudio están muy fragmentadas, constituye un importante refugio para aves residentes y migratorias.

Palabras clave: abundancia, bosque ribereño, categorías tróficas, diversidad, gremios tróficos, riqueza.

\section{ABSTRACT}

The composition and structure of avian assemblage were analyzed at four localities located on the banks of Maimón River: confluencia Maimón river with Yuna river, highway Maimon-Piedra Blanca, Piedra Blanca y Los Plátanos. A total of 598 individuals belonging to 51 species and 28 families of birds were recorded. The Maimón-Piedra Blanca locality have registered the highest values of the Shannon index with $\mathrm{H}^{`}=3.36$, it was also the station with the highest avian abundance and species richness with 217 individuals and 43 species respectively. 
In contrast, the station located in Los Plátanos presented the lowest diversity and species richness with $\mathrm{H}^{`}=2.92$ and 29, respectively, and the lowest abundance with 118 individuals. Seven trophic guilds and 15 trophic categories were registered. The carnivores and omnivores were the most diverse and abundant guilds. Significant differences were recorded on the relative abundance of birds by trophic guilds and between the studies areas $\left(\mathrm{X}^{2}=43.32, \mathrm{df}=18, \mathrm{p}=<0.01\right)$. Specie richness by trophic guild were not statistically different among studies areas $\left(\mathrm{X}^{2}=6.67 \mathrm{df}=18, \mathrm{p}=0.99\right)$. Significant differences were not recorded between trophic category by studies areas $\left(\mathrm{X}^{2}=39.34\right.$, $\left.\mathrm{df}=42, \mathrm{p}=0.58\right)$. Our results suggested that the avian assemblage associated at the Maimón River is equitable distributed. In addition, the bird's assemblage was mainly composed for wide-ranging and habitat generalist's species. Finally, although the studies areas were highly fragmented, they are an important refuge for resident and migratory birds.

Keywords: abundance, riparian forest, trophic categories, species diversity, trophic guilds, richness.

\section{INTRODUCCIÓN}

Los bosques ribereños se caracterizan por estar formados por un cuerpo de agua con vegetación asociada a sus márgenes, y constituyen zonas ecotonales, es decir, son áreas de transición entre los ambientes acuáticos y la vegetación circundante (Gómez et al., 2016). Estos bosques se distribuyen en franjas delgadas, debido a esto son muy escasos y sensibles a los disturbios antropológicos (Kauffman y Krueger, 1984; Arcos-Torres et al., 2008; Gómez et al., 2016). A pesar de esto, los bosques ribereños destacan por presentar una diversidad biológica única y de alta importancia. Entre la biodiversidad presente en estos ambientes se encuentra una alta diversidad de plantas y animales silvestres, así como una alta variedad de hábitats, lo cual favorece el establecimiento de especies especialistas de bosque húmedo (Arcos-Torres et al., 2008).

Los bosques ribereños presentes en áreas productivas, donde la agricultura y la ganadería son los principales usos de la tierra, funcionan como corredores biológicos, al permitir el desplazamiento y flujo genético entre parches remanentes de la vegetación nativa, y a veces como único hábitat para la fauna silvestre (Arcos-Torres, 2005; Nores et al., 2005; Arcos-Torres et al., 2008). Estos bosques sirven como refugio de alimentación y de reproducción para una gran variedad de fauna, además sirven como agentes reguladores de contaminantes y nutrientes que provienen de zonas cercanas (Gómez et al., 2016). Adicionalmente, son más complejos que los ambientes que los rodean, tanto estructural como funcionalmente, favoreciendo de esta forma una mayor riqueza y abundancia, lo cual está asociado a la disponibilidad del recurso agua, complejidad de hábitat, alta disponibilidad de recursos alimenticios y beneficios asociados a los efectos de borde (Rosetti y Giraudo, 2003; López-Barrera, 2004; Peña-Becerril et al., 2005; Palmer y Bennett, 2006; Guichón y Cassini, 2007; Arcos-Torres et al., 2008).

En el caso de la avifauna asociada a los bosques ribereños, numerosos estudios han documentado la importancia de estos ambientes para las poblaciones de aves (Arcos, 2005; Nores et al., 2005; Palmer y Bennett, 2006; Arcos-Torres et al., 2008;). Palmer y Bennett (2006) encontraron que la composición poblacional de las aves en estos ecosistemas es de mayor riqueza, diversidad y abundancia que en áreas vecinas. Gómez et al. (2016), resaltan la importancia de estos bosques como refugio para las aves migratorias y residentes. Además, Arendt et al. (2012), destacan la relevancia de estos ambientes como refugio para aves frugívoras, insectívoras y nectarívoros, así como para aves dependientes de estos hábitats. 
Con base a este contexto, el presente trabajo pretende aportar información acerca de algunos aspectos ecológicos de la avifauna asociada a cuatro localidades ubicadas en la ribera del río Maimón, específicamente, se persigue conocer cómo se estructura y compone el ensamblaje de aves asociado a los bosques ribereños de este río. También, se busca generar información que sirva como línea base para la realización de estudios diversos sobre el ensamblaje de aves asociadas a este ecosistema.

\section{OBJETIVOS}

- Analizar la estructura y composición del ensamblaje de aves presente en cuatro localidades del río Maimón, República Dominicana.

\section{MATERIALES Y MÉTODOS}

Área de estudio. El río Maimón se localiza entre las provincias Monseñor Nouel y Sánchez Ramírez, en la región norte de República Dominicana. Este río nace en el parque nacional Loma la Humeadora, en la provincia Monseñor Nouel y se extiende a través de los municipios de Piedra Blanca y Maimón, hasta su confluencia con el río Yuna en la provincia Sánchez Ramírez (Fig. 1). Posee una extensión de $33.62 \mathrm{~km}$ de longitud y cubre un área de $99.49 \mathrm{~km}^{2}$ (Torres-Pineda, 2019).

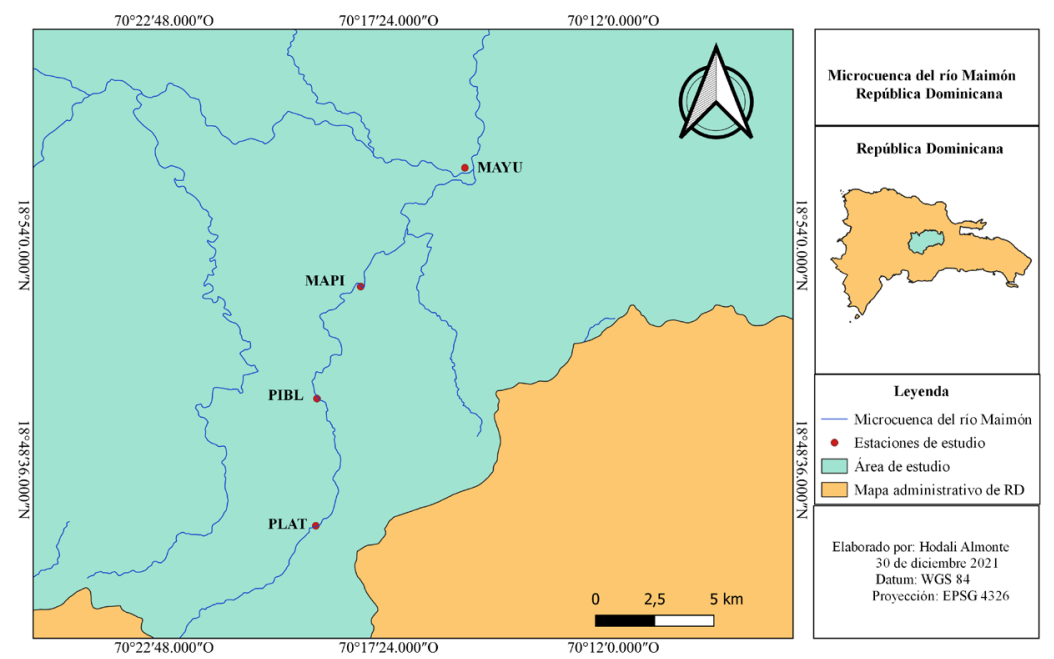

Figura 1. Localización del área de estudio. MAYU = confluencia del río Maimón con río Yuna; MAPI = carretera Maimón-Piedra Blanca; PIBL = Piedra Blanca; PLAT = Los Plátanos.

Dentro de los bosques asociados a este río se estudiaron cuatro estaciones ubicadas en las comunidades: la confluencia del río Maimón con río Yuna (MAYU), carretera Maimón Piedra Blanca (MAPI), Piedra Blanca (PIBL), y Los Plátanos (PLAT). La estación MAYU se ubica el curso bajo del río Maimón, en la zona de influencia de los ríos Maimón y Yuna, la cual es una extensa área de tierras destinada a la agricultura, específicamente al cultivo de plátano (Musa paradisiaca) y guineo (Musa sapientum), así como a la ganadería. La estación PIBL corresponde al curso medio-alto del río Maimón, ubicada en el área urbana del municipio Piedra Blanca (Fig. 2). Esta estación constituye una delgada franja de bosque de menos de 30 metros de ancho, impactada por asentamientos humanos en su ribera, áreas agropecuarias y el paso de 
caminos vecinales. En el caso de la estación MAPI, está se ubica en el curso medio-bajo del río. Se caracteriza por presentar un importante remanente del bosque ribereño original con parches de bosques que se extienden a más de $1.5 \mathrm{~km}$ y un ancho de franja de más de $50 \mathrm{~m}$ de longitud, a este remanente de bosque se asocian cultivos como el cacao (Teobroma cacao) y frutales de mandarina (Citrus reticulata) y naranja dulce (Citrus sinensis), (Torres-Pineda, 2019) (Fig. 2). Finalmente, la estación PLAT se ubica en la comunidad Los Plátanos y se encuentra ubicada en el curso alto del río Maimón, en el distrito municipal de Juan Adrián, paraje Los Plátanos. Al igual que en la estación PIBL, el área muestreada constituye una delgada franja de bosque de menos 20 metros de ancho, impactada por asentamientos humanos en su ribera, áreas agropecuarias y el paso de caminos vecinales y carretera.
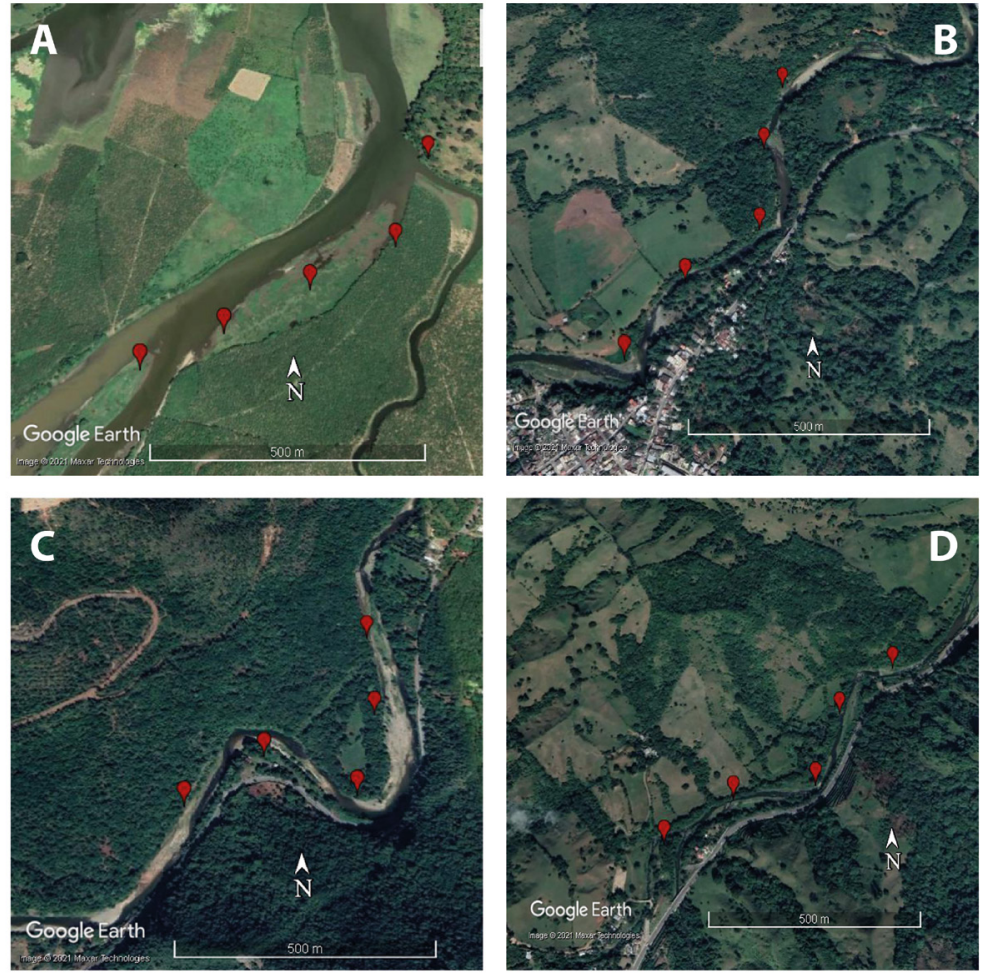

Figura 2. Imágenes satelitales de las estaciones de muestreo. A = confluencia del río Maimón con río Yuna; $\mathrm{B}=$ carretera MaimónPiedra Blanca; $\mathrm{C}=$ Piedra Blanca; $\mathrm{D}=$ Los Plátanos. Símbolos rojos $=$ estaciones de muestreos.

Métodos. Con el objetivo de caracterizar el ensamblaje de aves se utilizó el método de transectos en franjas (Ralph et al., 1995, 1996). Este método se caracteriza porque se aplica en línea recta divididas en estaciones de diferentes longitudes y tiene la particularidad de que el observador registra las aves detectadas mientras camina (Ralph et al., 1995, 1996). En cada una de las localidades de estudio se estableció un transecto de $1 \mathrm{~km}$; cada uno fue dividido en cinco estaciones de 200 metros de longitud y 50 metros de ancho (Fig. 2). Se registraron todos los individuos observados o escuchados durante un lapso de 60 minutos por transectos (Ralph et al., 1995, 1996).

Los muestreos se realizaron entre los meses de diciembre del 2016 y mayo del 2017. El levantamiento de la información de campo se efectuó entre las $0600 \mathrm{~h}$ y las $1000 \mathrm{~h}$ y entre las $1600 \mathrm{~h}$ y $1800 \mathrm{~h}$. En cada una de las estaciones se realizaron dos muestreos, durante dos días seguidos. La identificación visual de las especies se realizó con binoculares Nikon 10 x 42 (10 de aumento y los objetivos de $42 \mathrm{~mm}$ de diámetro), siguiendo a Latta et al. (2006). 
Para la organización y el ordenamiento taxonómico se siguió el sistema de clasificación The North American Classification Committee (NACC, Chesser et al., 2020).

Análisis de los datos. La abundancia relativa de las especies presentes en cada transecto fue estimada dividiendo el número de individuos de cada especie entre el número total de individuos registrado por transecto y multiplicado por 100 , mediante la fórmula $\mathrm{P}_{\mathrm{i}}=\left(\mathrm{ns}_{\mathrm{i}} / \mathrm{\Sigma} \mathrm{n}\right) \mathrm{x} 100$; donde $\mathrm{P}_{\mathrm{i}}$, representa la abundancia relativa de las especies presentes en las zonas de estudio, ns $\mathrm{i}_{\mathrm{i}} \mathrm{el}$ número de individuos de cada especie i y $\Sigma$ n la sumatoria del número total de individuos (Pettingill, 1969). Adicionalmente, se siguió a Salgado-Ortiz (1999), para establecer cuatro categorías de abundancia: especies raras (0.08-1.9), especies poco común (2.0-4.9), especie común (5.0-9.9) y abundante (10-24.5).

Para conocer mejor la ecología de las comunidades de aves estudiadas, se estimaron varios índices de diversidad y equidad de especies: índice de diversidad de Shannon $\left(\mathrm{H}^{\prime}\right)$, índice de Equidad de Pielou (J'), índice de Dominancia de Simpson $(\lambda)$ y recíproco de Simpson (D-1), (Moreno, 2001). Con el objetivo de conocer los gremios alimentarios de las comunidades de aves en estudio, las especies registradas fueron agrupadas en gremios y categorías tróficas de acuerdo con su dieta y sustrato de forrajeo, siguiendo a González-Salazar et al. (2014); estos mismos autores evaluaron 25 categorías tróficas, sin embargo, para fines de esta investigación solo se evaluaron 15 categorías debido a que en algunos casos estas se solapaban o no tenían representantes dentro de las comunidades de aves estudiada.

Mediante la prueba de Chi-cuadrado se determinó que tan similar fue la estructura trófica de las comunidades evaluada. Tanto los análisis de diversidad como la estructura trófica del ensamblaje de aves fueron analizados con el software Past 4.06b (Hammer et al., 2001).

\section{RESULTADOS Y DISCUSIÓN}

Abundancia. Se registró un total de 598 individuos, pertenecientes a 28 familias, 48 géneros y 51 especies de aves (Tabla I). Las familias con mayor riqueza de especies fueron Parulidae (7 especies: $14 \%$ ), Ardeidae (6 especies: $11.76 \%$ ) y Columbidae (4 especies: 7.84 \%). La abundancia absoluta de individuos varió por estación encontrándose que la estación próxima a la carretera Maimón Piedra Blanca (MAPI) registró la mayor abundancia de individuos con 217, mientras que la estación ubicada en la comunidad de Los Plátanos (PLAT) presentó la menor, con 120 individuos (Tabla I). La riqueza de especies observadas fue más alta para la estación MAPI, con 43 especies, a diferencia de la estación PLAT que reportó los valores más bajos con 29 (Tablas I y II).

A pesar de que las áreas estudiadas corresponden a franjas estrechas de bosques ribereños conectados a agropaisajes, estas conservan una alta riqueza de aves, con 51 especies, lo cual constituye aproximadamente el $17 \%$ del total de especies reportadas para la isla, que ha sido 300 (Latta et al., 2006; Latta y Wallace, 2012). También, incluye algunas especies amenazadas para República Dominicana como: la perdiz colorada (Geotrygon montana) y el carrao (Aramus guarauna), ambas especies amenazadas por factores como la cacería ilegal, depredación por especies introducidas y pérdida de sus hábitats (Latta et al., 2006). Además, destaca la presencia en estas áreas de la cigüita del río (Parkesia motacilla), la cual es considerada como de importancia para la conservación, debido a que es un buen indicador de la salud de los sistemas dulceacuícolas (Mattsson y Cooper, 2006; Almonte-Espinosa y Latta, 2011). 


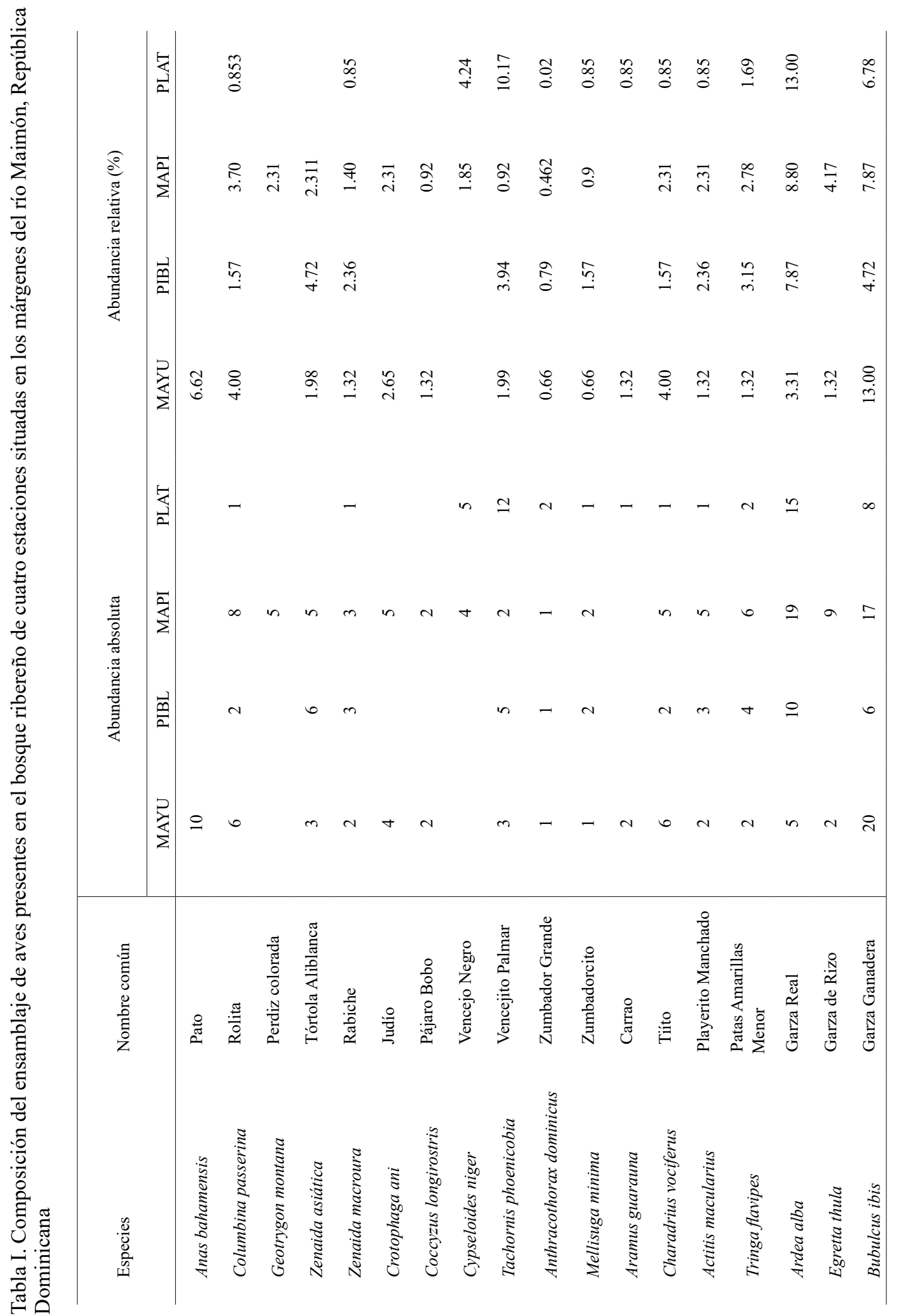




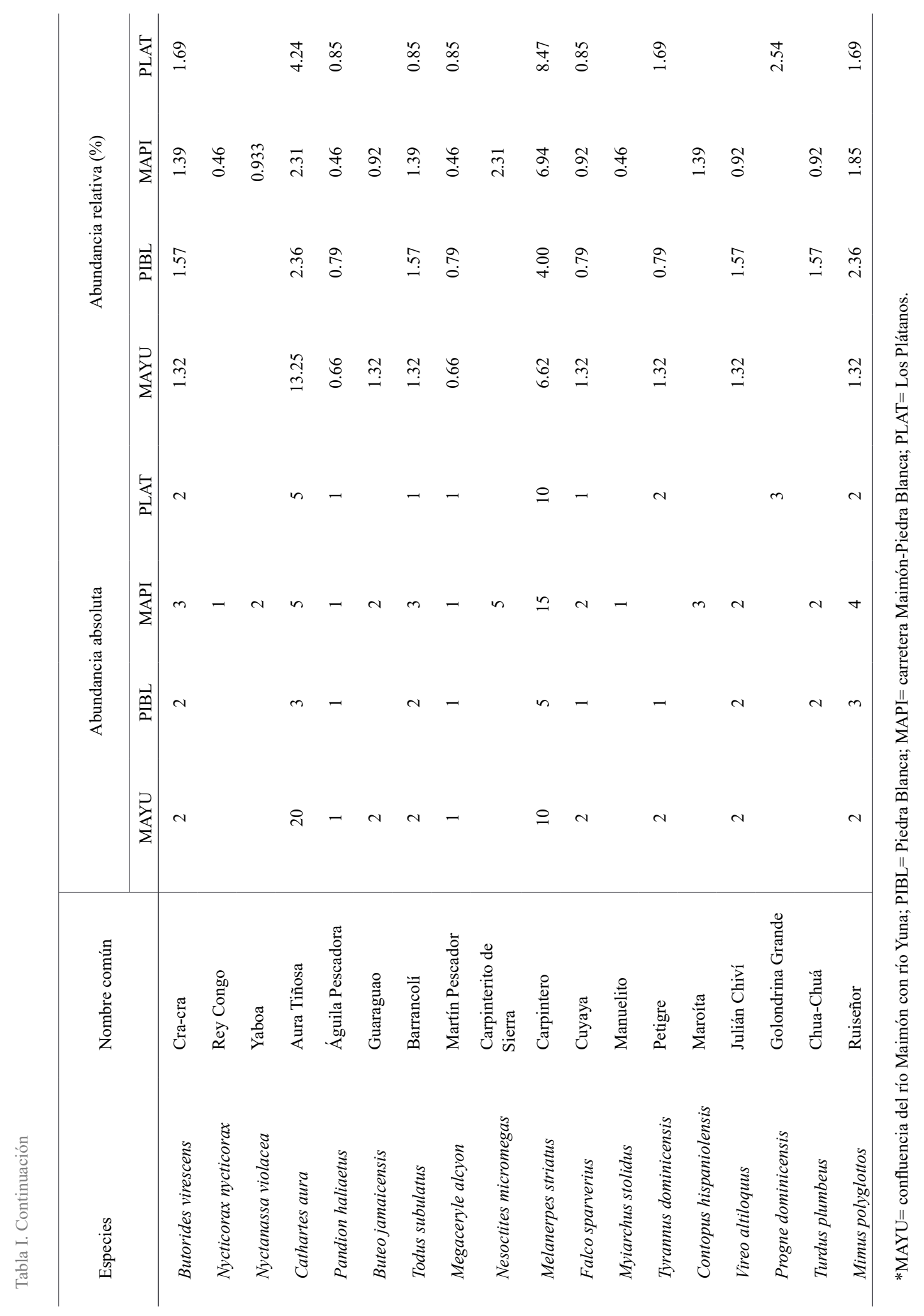




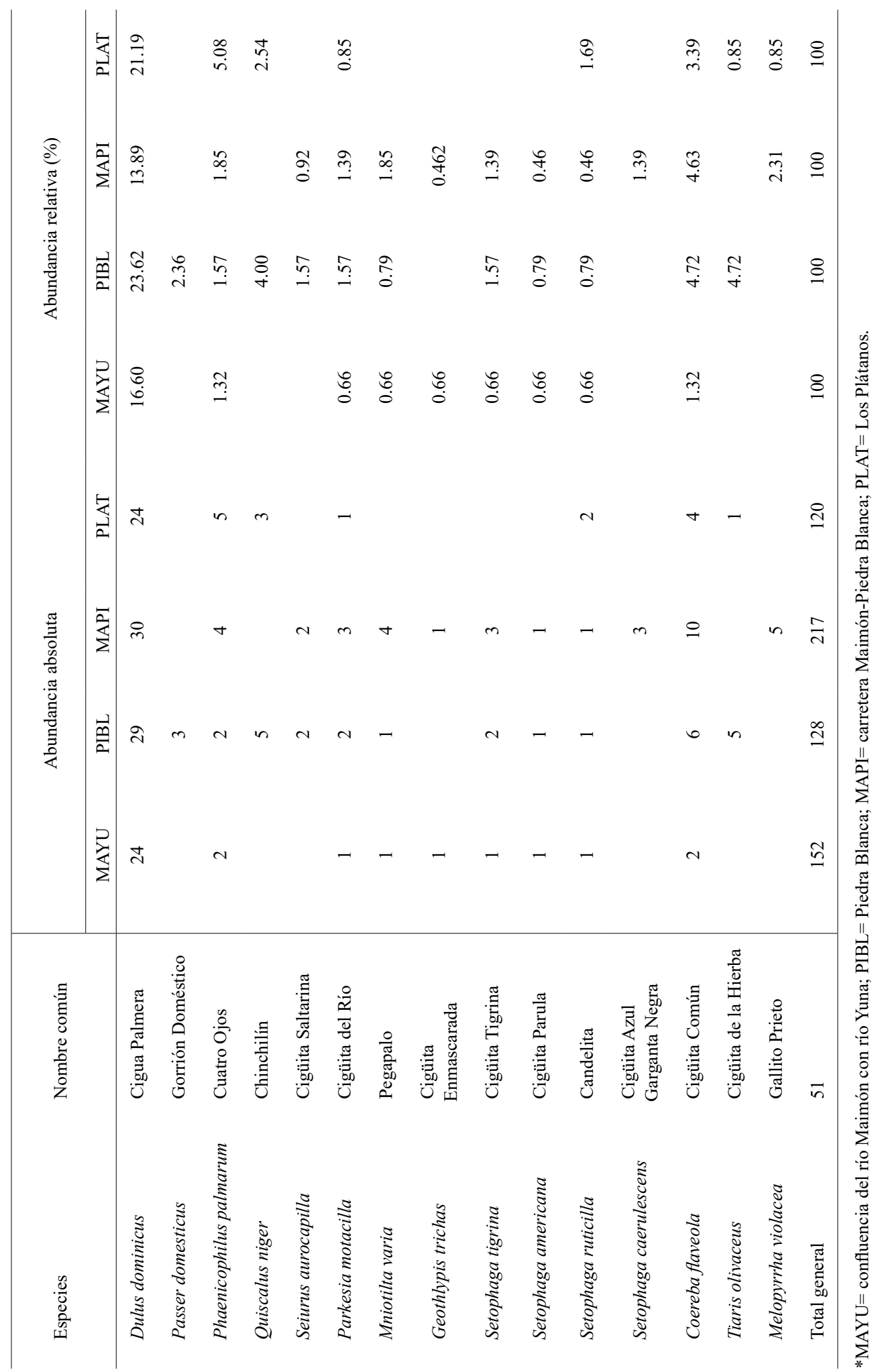


Los valores de riqueza observada y de abundancia registrada (Tablas I y II), indican que estas áreas presentan una alta riqueza de especies y abundancia de aves. Estos resultados concuerdan con lo reportado en varios estudios realizados en los trópicos, los cuales destacan la importancia de la vegetación ribereña para las poblaciones de aves (Lock y Naiman, 1998; Skagen et al., 1998), notando que la composición poblacional de aves en estos ecosistemas es de mayor riqueza y abundancia de especies que en áreas vecinas a los bosques ribereños, sin embargo, estas diferencias pueden variar mucho, según el contexto del paisaje en que se encuentren y las características del área misma (Lock y Naiman, 1998; Skagen et al., 1998). Un ejemplo de esto es lo observado en las estaciones de estudio, donde se registró que la riqueza y abundancia de aves variaron según el grado de transformación de los ambientes, para citar un ejemplo, la estación MAPI presentó la mayor riqueza y abundancia de aves (Tablas I y II), posiblemente debido a que esta área presenta un importante remanente de la vegetación nativa, cuyo ancho de franja es mayor $50 \mathrm{~m}$ de ancho a ambos lados del río. En el lado opuesto tenemos la estación PLAT, la cual mostró una menor abundancia y riqueza, probablemente esto se relaciona con el grado de fragmentación que se registra en esta estación, ya que el bosque ribereño se encuentra muy impactado por la presencia de asentamientos humanos, infraestructura de carretera y actividades agropecuarias.

La abundancia relativa fue similar por estación, encontrándose que la cigua palmera (Dulus dominicus), fue la especie más abundante en las cuatro estaciones (MAYU $=16.55 \%$ PIBL $=24.59 \%$; MAPI $=12.07 \%$; PLAT $=12.71 \%$ ), (Tabla II). Seguido del carpintero (Melanerpes striatus) con $(\mathrm{MAYU}=6.62 \%$; PIBL $=6.55 \%$; MAPI $=6.76 \%$; PLAT $=8.47 \%)$ y la garza real $($ Ardea alba $)(\mathrm{MAYU}=4.63 \%$; PIBL $=4.09 \%$; MAPI $=3.86 \%$; PLAT $=12.71 \%)$. De acuerdo con las categorías de abundancias evaluadas, solo la cigua palmera puede ser considerada como una especie abundante en las cuatro estaciones de estudio, debido a que su abundancia relativa se encontró dentro del rango 10-24.5\% del total de aves registradas (Tabla I), mientras que el carpintero fue una especie común, con rango entre 5.0-9.9\% de abundancia relativa, en las cuatro áreas (Tabla I).

En ese mismo sentido, la abundancia relativa de algunas especies varió por estación, por ejemplo, la garza real y la garza ganadera fueron abundantes en la estación MAYU y la estación MAPI, en que la abundancia relativa fue de $13 \%$ para ambas estaciones, mientras que en las estaciones de PIBL y PLAT estas especies son consideradas como comunes y poco comunes, con rango de abundancia entre 2.0-4.9 y 5.0-9.9 \% (Tabla I). Finalmente, se encuentran especies con abundancia relativa entre los rangos $0.08-1.9 \%$ consideradas como raras para esta investigación por los valores de abundancia relativa registrados (Tabla I).

La alta abundancia de especies, como la cigua palmera y el carpintero se debe a sus hábitos de vida ya que, probablemente, son las aves de mayor distribución y abundancia en la isla (Latta et al., 2006); además, tienden a ser abundantes en áreas agropecuarias, donde hay plantas de palmeras, frutales y pequeños remanentes de bosques o matorrales. Estos son utilizados por ambas especies para anidar y forrajear, ya que generalmente son fuentes importantes de frutas e insectos (Arcos-Torres, 2005; Arcos et al., 2008; Gómez et al., 2016). En el caso de la garza real, es importante aclarar que esta especie es dependiente de ambientes ribereños o acuáticos, debido a que se alimenta de una amplia variedad de macroinvertebrados y vertebrados asociados a cuerpos de agua, lo cual favorece la presencia y abundancia de esta especie en ambientes ribereños (Arendt et al., 2012; McCrimmon et al., 2020). En cambio, las aves reportadas como raras corresponden a especies de hábitos específicos y que por lo general suelen ser afectadas por la fragmentación y transformación de hábitats, como es el caso de Myiarchus stolidus (Latta et al., 2006; Arcos-Torres et al., 2008; Joseph, 2020), (Tabla I). 
Diversidad. La diversidad de especies varió ligeramente por estación, encontrándose que las estaciones de la carretera río Maimón-Piedra Blanca y Piedra Blanca mostraron los valores más altos del índice de Shannon: $\mathrm{H}^{\prime}=3.36 \mathrm{y} \mathrm{H}^{\prime}=3.03$. La estación ubicada en la carretera Maimón-Piedra Blanca presentó el valor más bajo de dominancia de especies $(\lambda=0.04)$, siendo su recíproco (D-1) 0.95 , mientras que la confluencia del río Maimón con río Yuna y la comunidad de Piedra Blanca presentaron el más alto $(\lambda=0.08)$ y su recíproco fue 0.92 (Tabla II). Se encontró que los valores de equidad de Pielou fueron más altos para la estación de la carretera Maimón-Piedra Blanca, $\mathrm{J}=0.90$, mientras que el valor más bajo lo registró la estación de la confluencia del río Maimón con río Yuna, $\mathrm{J}=0.83$. Los índices de diversidad analizados indican que la comunidad de aves en las cuatro localidades de estudio se distribuye de forma equitativa, lo cual se puede apreciar tanto en los valores del índice de Shannon y como del índice de equidad de Pielou (Tabla II). Sin embargo, a pesar de los altos valores de diversidad registrados de acuerdo con el índice de dominancia de Simpson y el índice de equidad de Pielou, estas comunidades se ven ligeramente afectadas por la dominancia de especies abundantes, como la cigua palmera (Dulus dominicus), el carpintero (Melanerpes striatus) y la garza real (Ardea alba) (Tablas I y II). La alta abundancia de estas especies es explicada por sus hábitos de vida y condiciones ambientales de los hábitats estudiados, los cuales favorecen su presencia y abundancia.

Tabla II. Datos ecológicos del ensamblaje de aves asociado a cuatro estaciones del río Maimón, República Dominicana

\begin{tabular}{l|cccc}
\hline & MAYU & PIBL & MAPI & PLAT \\
\hline Riqueza observada & 37 & 31 & 43 & 29 \\
Índice de entropía de Shannon & 2.97 & 3.03 & 3.36 & 2.92 \\
Equidad de Pielou & 0.83 & 0.87 & 0.90 & 0.87 \\
Dominancia Simpson & 0.08 & 0.08 & 0.04 & 0.07 \\
Recíproco de Simpson & 0.92 & 0.92 & 0.95 & 0.93 \\
\hline
\end{tabular}

*MAYU = confluencia del río Maimón con río Yuna; PIBL = Piedra Blanca; MAPI = carretera Maimón-Piedra Blanca; PLAT $=$ Los Plátanos.

Gremios tróficos. Las especies registradas fueron agrupadas en siete gremios tróficos y 15 categorías tróficas. Los gremios tróficos encontrados fueron: carnívoros, frugívoros, granívoros, insectívoros, nectarívoros, carroñeros y omnívoros (Tablas III y IV, Figs. 3 y 4). Los carnívoros fueron el grupo más diverso, con 15 especies, siendo los carnívoros acuáticos los mejor representados con 8 especies (Fig. 2), seguido de los omnívoros, con 14 especies, de las cuales 12 son omnívoros arbóreos. Luego, tenemos los insectívoros con diez especies, siendo los insectívoros excavadores y recolectores de superficies los más diversos, con tres especies (Tabla I). La alta variedad de gremios sugiere que tanto la composición como estructura trófica de estas comunidades de aves, podría ser atribuido al efecto de borde que tienen los ecosistemas 
ribereños, debido a su forma lineal y estrecha (Marateo y Arturi, 2013; Núñez e Infante-Rivero, 2019), pudiendo beneficiarse de los diferentes recursos alimenticios que ofrecen los ambientes ribereños, como lo son: una gran diversidad de macroinvertebrados acuáticos, pequeños vertebrados y de frutos, semillas, insectos y otros invertebrados asociados a la vegetación ribereña que se extiende a lo largo de las zonas evaluadas (Núñez e Infante-Rivero, 2019).

La alta riqueza de especies en el gremio de los carnívoros, se asocia a que los ambientes ribereños proporcionan una amplia variedad de hábitats, debido a que son más complejos, tanto de forma estructural como funcional que los ambientes que los rodean, lo que favorece una mayor riqueza y abundancia de aves, lo cual está relacionado con la disponibilidad del recurso agua, complejidad de hábitats, alta disponibilidad de recursos alimenticios (Rosetti y Giraudo, 2003; López-Barrera, 2004; Guichón y Cassini, 2007; Palmer y Bennett, 2006; Arcos-Torres et al., 2008; Salas-Correa y Mancera-Rodríguez, 2018). Además, la conexión de las áreas de estudio con agropaisajes favorece el establecimiento de especies oportunistas, como son los miembros del gremio de los carnívoros, los cuales ocupan una amplia variedad de nichos. Por ejemplo, la cuyaya (Falco sparverius) y el guaraguao (Buteo jamaicensis) son carnívoros generalistas que cazan al vuelo o en los árboles de áreas abiertas, mientras que por otro lado, tenemos carnívoros dependientes de cuerpos de agua, como el guincho (Pandion haliaetus) y las garzas: garza de rizos (Egretta thula), garza real (Ardea alba) y el rey congo (Nyctanassa violacea), los cuales consumen una amplia variedad de organismos acuáticos, tales como peces, anfibios pequeños y macroinvertebrados. Adicionalmente, se pueden mencionar la garza ganadera (Bubulcus ibis), la cual es un ave asociada a zonas agropecuarias, que se alimenta de invertebrados y pequeños vertebrados.

Se registraron diferencias significativas en la abundancia relativa de aves por gremios $\left(\mathrm{X}^{2}=43.32, \mathrm{df}=18, \mathrm{p}=<0.01\right)$, (Tabla III; Figs. 3 y 4). Encontrándose que las aves omnívoras fueron el gremio trófico más abundante en las cuatro estaciones de estudio, así como el segundo grupo en diversidad de especies, debido a que estas aves generalmente se asocian a zonas agrícolas y ganaderas, ya que estos hábitats presentan una gran variedad de recursos de alimentación, por lo que se ven beneficiadas por la fragmentación del ecosistema (López-Barrera, 2004; Mastrangelo y Gavin, 2012). Otro factor que beneficia la abundancia y diversidad del gremio de los omnívoros es que aprovechan pequeñas franjas de vegetación ribereña asociadas a las áreas productivas para forrajear y anidar (Arcos-Torres et al., 2008; Mastrangelo y Gavin, 2012). Esta tendencia se puede observar en las áreas evaluadas, ya que en todos los casos la vegetación remanente corresponde a franjas de bosques ribereños fragmentadas que se conectan con agropaisajes o zonas suburbanas.

No se encontró diferencia en la riqueza de especies por gremio trófico $\left(\mathrm{X}^{2}=6.67\right.$, $\mathrm{df}=18, \mathrm{p}=0.99$ ). Tampoco se encontraron diferencias significativas por categorías tróficas en las zonas evaluadas $\left(\mathrm{X}^{2}=39.34, \mathrm{df}=42, \mathrm{p}=0.58\right)$. La similitud en la composición de especies por gremio trófico se relaciona con el grado de fragmentación que presentan los hábitats analizados, específicamente a que las estaciones de estudio presentan grados de fragmentación similares, por ejemplo, tanto la estación PIBL como la estación PLAT presentan áreas de cultivos y asentamientos humanos en su periferia, mientras que las estaciones MAYU y MAPI (Fig. 2), se encuentran en áreas agropecuarias, con la salvedad de que la estación MAPI conserva un importante remanente de la vegetación nativa o típica de ambientes ribereños mezclada con plantaciones de cacao y frutales, lo cual favorece una alta riqueza de especies y abundancia de aves dependientes de cuerpos de agua y diversas especies de bosque, como los insectívoros, frugívoros, nectarívoros y omnívoros. 


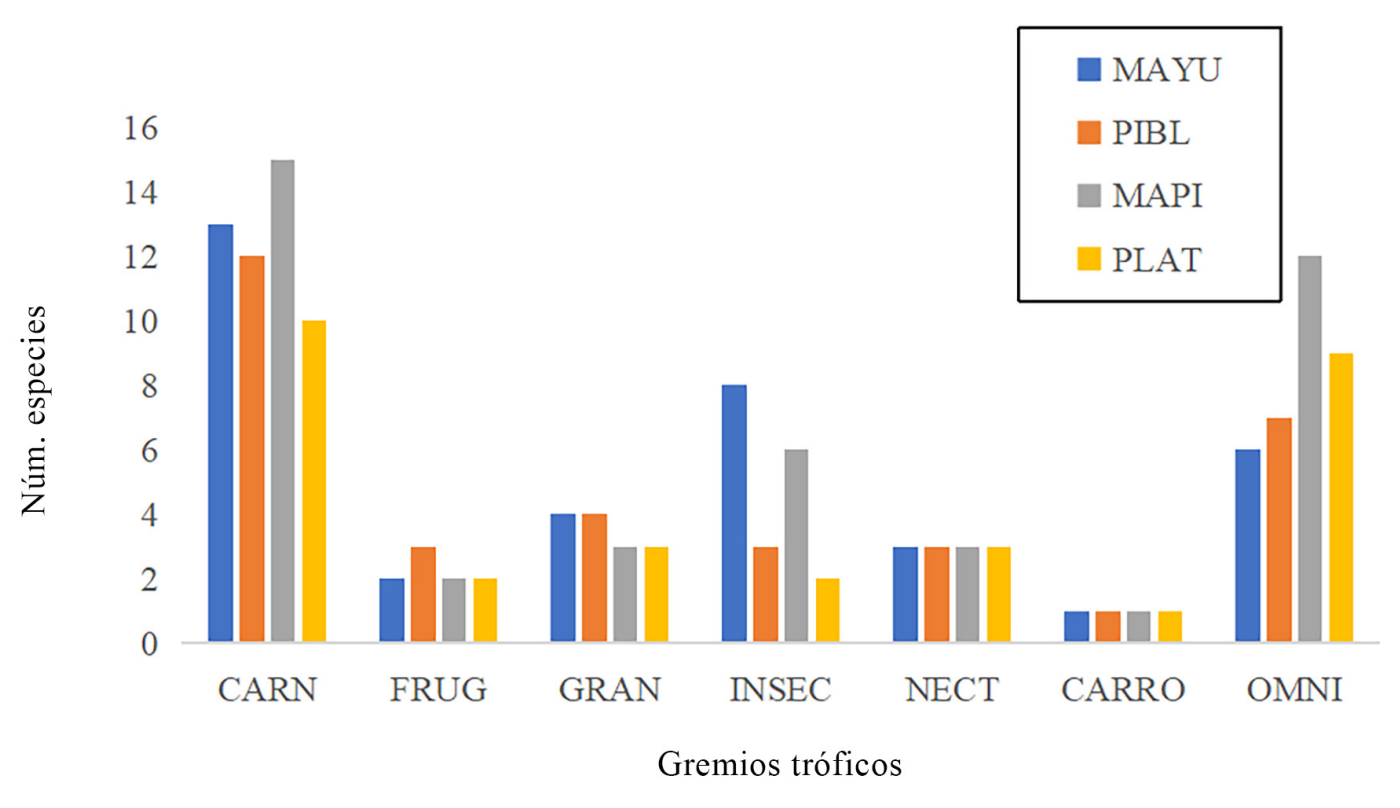

Figura 3. Estructura trófica del ensamblaje de aves presente en cuatro estaciones del río Maimón, República Dominicana. MAYU = confluencia del río Maimón y río Yuna; PIBL = comunidad de Piedra Blanca; MAPI = carretera Maimón-Piedra Blanca; PLAT $=$ Los Plátanos. CARN = carnívoros; FRUG = frugívoros; GRAN = granívoros; INSEC $=$ insectívoro; $\mathrm{NECT}=$ nectarívoro; $\mathrm{CARRO}=$ carroñeros; OMNI= omnívoros.

Tabla III. Estructura trófica del ensamblaje de aves estudiado en cuatro estaciones del río Maimón, República Dominicana

\begin{tabular}{|c|c|c|c|c|c|c|c|c|c|c|c|c|}
\hline \multirow{2}{*}{$\begin{array}{l}\text { GREMIO } \\
\text { TRÓFICO }\end{array}$} & \multicolumn{4}{|c|}{ Núm. especies } & \multicolumn{4}{|c|}{ Abundancia de individuos } & \multicolumn{4}{|c|}{ Abundancia relativa $(\%)$} \\
\hline & MAYU & PIBL & MAPI & PLAT & MAYU & PIBL & MAPI & PLAT & MAYU & PIBL & MAPI & PLAT \\
\hline Carnívoros & 13 & 12 & 15 & 10 & 26 & 20 & 40 & 20 & 17.20 & 15.74 & 23.15 & 16.95 \\
\hline Frugívoros & 2 & 3 & 2 & 2 & 20 & 30 & 30 & 15 & 12.30 & 23.42 & 13.88 & 12.71 \\
\hline Granívoros & 4 & 4 & 3 & 3 & 16 & 11 & 30 & 2 & 10.60 & 9.00 & 15.30 & 1.69 \\
\hline Insectívoros & 8 & 3 & 6 & 2 & 25 & 20 & 40 & 34 & 17.00 & 15.73 & 13.88 & 28.81 \\
\hline Nectarívoros & 3 & 3 & 3 & 3 & 4 & 9 & 13 & 7 & 2.70 & 7.00 & 4.63 & 5.93 \\
\hline Carroñeros & 1 & 1 & 1 & 1 & 20 & 3 & 5 & 10 & 13.20 & 2.34 & 2.31 & 8.48 \\
\hline Omnívoros & 6 & 7 & 12 & 9 & 40 & 30 & 58 & 30 & 26.50 & 26.77 & 26.85 & 25.43 \\
\hline
\end{tabular}

*MAYU = confluencia del río Maimón con río Yuna; PIBL = Piedra Blanca; MAPI = carretera Maimón-Piedra Blanca; PLAT = Los Plátanos. 
Los gremios insectívoros, frugívoros y nectarívoros estuvieron presentes en menor proporción (Tabla III), debido posiblemente a que son especies con requerimientos específicos de hábitats y se asocian con una mayor heterogeneidad y mejor calidad ecológica, por lo tanto, su riqueza y abundancia dependen de las condiciones de conservación de los hábitats (Shahabuddin y Kumar, 2006; Reales et al., 2009; Ribeiro y Melo, 2012). Por ejemplo, los insectívoros fueron el tercer grupo más diverso y abundante, sin embargo, las especies registradas son aves de amplia distribución que tienden a ser abundantes en zonas abiertas y dependientes de cuerpos de agua, que aprovechan franjas de bosques ribereños para forrajear en los bordes de la vegetación y ambiente acuático.

Los granívoros, a pesar de ser reconocidos en la literatura como un grupo abundante en áreas agrícolas y ganaderas, ocuparon el cuarto lugar en abundancia, lo que difiere de lo encontrado por Chace y Walsh (2004), Reales et al. (2009) y Ding et al. (2015), quienes sugieren que los ambientes fragmentados tienden a favorecer estas especies. Una posible explicación es que estas aves tienden a ocupar áreas más abiertas y utilizan los bosques ribereños de forma ocasional, debido a que en estas áreas las semillas no son tan abundantes. En este mismo sentido, la baja abundancia y riqueza del gremio de los carroñeros, se explica debido a que en la isla el aura tiñosa (Cathartes aura), es el único miembro de dicho gremio y hasta cierto punto fue una especie abundante por lo menos para las estaciones adyacentes a zonas ganaderas como fueron las estaciónes de la confluencia del río Maimón con río Yuna y Los Plátanos (Tablas I y III).

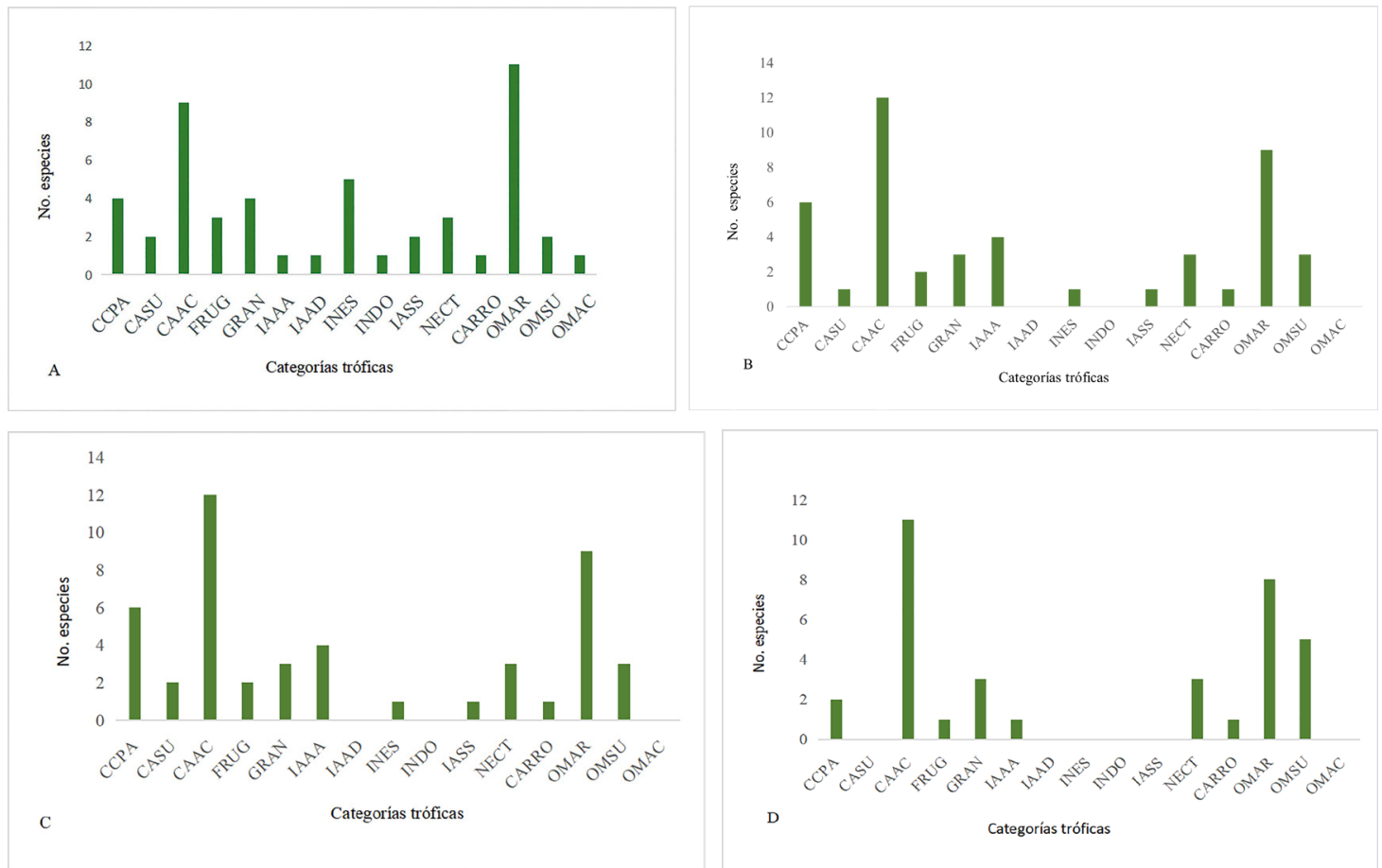

Figura 4. Categorías tróficas del ensamblaje de aves presente en cuatro localidades del río Maimón. $\mathrm{A}=$ confluencia del río Maimón con río Yuna; $\mathrm{B}=$ Piedra Blanca; $\mathrm{C}=$ carretera Maimón-Piedra Blanca; $\mathrm{D}=$ Los Plátanos. CCPA $=$ carnívoros cazadores de presas que se alimentan al vuelo y presas arbóreas; CASU = carnívoros de suelo; CAAC = carnívoros acuáticos; FRUG = frugívoros; GRAN = granívoros; IAAA = insectívoros que se alimentan en el aire; IAAD = insectívoros que se alimentan sobre el dosel; INES = insectívoros excavadores, recolectores de insectos en superficie de hojas y troncos; INDO = insectívoros de suelo; IASO = insectívoros de subdosel y sotobosque; NECT = nectarívoros; CARRO = carroñeros; OMAR = omnívoros arbóreos; OMSU = omnívoros de suelo; OMAC = omnívoros acuáticos. 
Tabla IV. Composición trófica del ensamblaje de aves presente en el bosque ribereño de cuatro estaciones situadas en los márgenes del río Maimón, República Dominicana

\begin{tabular}{|c|c|c|}
\hline Especies & Nombre común & Categoría trófica \\
\hline Anas bahamensis & Pato & OMAC \\
\hline Columbina passerina & Rolita & OMSU \\
\hline Geotrygon montana & Perdiz Colorada & OMSU \\
\hline Zenaida asiatica & Tórtola Aliblanca & OMSU \\
\hline Zenaida macroura & Rabiche & GRAN \\
\hline Crotophaga ani & Judío & OMAR \\
\hline Coccyzus longirostris & Pájaro Bobo & CCPA \\
\hline Cypseloides niger & Vencejo Negro & INAR \\
\hline Tachornis phoenicobia & Vencejito Palmar & INAR \\
\hline Anthracothorax dominicus & Zumbador Grande & NECT \\
\hline Mellisuga minima & Zumbadorcito & NECT \\
\hline Aramus guarauna & Carrao & CAAC-CASU \\
\hline Charadrius vociferus & Tiíto & CASU \\
\hline Actitis macularius & Playerito Manchado & CAAC \\
\hline Tringa flavipes & Patas Amarillas Menor & CAAC \\
\hline Ardea alba & Garza Real & CAAC \\
\hline Egretta thula & Garza de Rizo & CAAC \\
\hline Bubulcus ibis & Garza Ganadera & CASU \\
\hline Butorides virescens & Cra-cra & CAAC \\
\hline Nycticorax nycticorax & Rey Congo & CAAC \\
\hline Nyctanassa violacea & Yaboa & CAAC \\
\hline Cathartes aura & Aura Tiñosa & CARR \\
\hline Pandion haliaetus & Águila Pescadora & CAAC \\
\hline Buteo jamaicensis & Guaraguao & CCPA \\
\hline Todus subulatus & Barrancolí & INES \\
\hline Megaceryle alcyon & Martín Pescador & CAAC \\
\hline Nesoctites micromegas & Carpinterito de Sierra & AMAR \\
\hline Melanerpes striatus & Carpintero & OMAR \\
\hline Falco sparverius & Cuyaya & CAAR \\
\hline Myiarchus stolidus & Manuelito & IAAD \\
\hline Tyrannus dominicensis & Petigre & IAAA \\
\hline Contopus hispaniolensis & Maroíta & IASO \\
\hline Vireo altiloquus & Julián Chiví & OMAR \\
\hline Progne dominicensis & Golondrina Grande & INES \\
\hline Turdus plumbeus & Chua-Chuá & OMSO \\
\hline Mimus polyglottos & Ruiseñor & OMAR \\
\hline
\end{tabular}


Tabla IV. Continuación

\begin{tabular}{|c|c|c|}
\hline Especies & Nombre común & Categoría trófica \\
\hline Dulus dominicus & Cigua Palmera & FRUG \\
\hline Passer domesticus & Gorrión Doméstico & GRAN \\
\hline Phaenicophilus palmarum & Cuatro Ojos & OMAR \\
\hline Quiscalus niger & Chinchilín & OMAR-OMSU \\
\hline Seiurus aurocapilla & Cigüita Saltarina & OMSO \\
\hline Parkesia motacilla & Cigüita del Río & CAAC \\
\hline Mniotilta varia & Pegapalo & IAAD \\
\hline Geothlypis trichas & Cigüita Enmascarada & CASU \\
\hline Setophaga tigrina & Cigüita Tigrina & OMAR-INAR \\
\hline Setophaga americana & Cigüita Parula & OMAR-INAR \\
\hline Setophaga ruticilla & Candelita & IAAA \\
\hline Setophaga caerulescens & Cigüita Azul Garganta Negra & INES-OMAR \\
\hline Coereba flaveola & Cigüita Común & NECT \\
\hline Tiaris olivaceus & Cigüita de la Hierba & GRAN \\
\hline Melopyrrha violacea & Gallito Prieto & FRUG \\
\hline Total general & 51 & 15 \\
\hline
\end{tabular}

*CCPA $=$ carnívoros cazadores de presas que se alimentan al vuelo y presas arbóreas; CASU = carnívoros de suelo; CAAC = carnívoros acuáticos; FRUG = frugívoros; GRAN = granívoros; IAAA = insectívoros que se alimentan en el aire; IAAD = insectívoros que se alimentan sobre el dosel; INES = insectívoros excavadores, recolectores de insectos en superficie de hojas y troncos; INDO = insectívoros de suelo; IASO = insectívoros de subdosel y sotobosque; NECT = nectarívoros; $\mathrm{CARRO}=$ carroñeros; OMAR $=$ omnívoros arbóreos; OMSU = omnívoros de suelo; OMAC = omnívoros acuáticos.

\section{CONCLUSIONES}

La estructura y composición del ensamblaje de aves presentes en los bosques ribereños asociados al río Maimón son dominados por especies de amplia distribución y generalistas de hábitats. Tanto la estructura como la composición de este ensamblaje es propiciada por las características de los ambientes evaluados, los cuales se encuentran muy fragmentados por las actividades agropecuarias y por la urbanización. A pesar de esto, las comunidades de aves presentes en las zonas de estudio se distribuyen de forma equitativa y presentan una alta riqueza de especies.

Los gremios tróficos más abundantes fueron los carnívoros y omnívoros, esto se debe a que los bosques ribereños, al ser áreas de transición o ecotonos, tienden a tener una alta variedad de hábitats; debido a esto brindan un mayor número de nichos y recursos para las especies dependientes de estos ecosistemas, lo que a su vez beneficia la presencia de diversos gremios tróficos y favorece la diversidad y abundancia de grupos oportunistas. Por ejemplo, los carnívoros ocuparon varios hábitats, como son ambientes acuáticos y áreas abiertas ubicadas en la periferia del bosque ribereño de las estaciones de estudio. Asimismo, las aves omnívoras fueron el segundo grupo más diverso y el de mayor abundancia, debido también a que la abundancia y riqueza de estas aves son favorecidas por los factores mencionados anteriormente, y porque los bosques ribereños son un excelente refugio de anidación y forrajeo. 
A pesar de que las áreas estudiadas presentan un alto grado de fragmentación y se conectan a agropaisajes, son un importante refugio para aves residentes y migratorias. Entre los grupos de aves favorecidos en estos ambientes se encuentran especies dependientes de cuerpos de agua como: Tringa flavipes, T. solitaria, Actitis macularius, Pandion haliaetus, Megaceryle alcyon y P. motacilla. Mientras que entre las especies de bosques se pueden mencionar: Setophaga caerulescens, Seiurus aurocapilla, Setophaga americana, Myiarchus stolidus, Todus subulatus y Geotrygon montana. La relevancia de estos ecosistemas para las aves se explica debido a que en zonas fragmentadas los bosques ribereños generalmente son pequeños parches, que constituyen el único sitio de anidación y forrajeo, debido también a la gran disponibilidad de recursos de hábitats y de alimentación que ofrecen para las comunidades de aves presentes.

Los resultados presentados en este trabajo son una aproximación al conocimiento de la avifauna asociada al río Maimón y una línea base para el estudio de diversos aspectos ecológicos, de comportamiento y biología del ensamblaje de aves presentes en este ambiente. Debido a esto, se recomienda realizar un mayor esfuerzo de muestreo, para conocer ampliamente cómo se estructura y está compuesto el ensamblaje de aves asociadas al río Maimón.

\section{AGRADECIMIENTOS}

A Cristian Marte, Katihusca Rodríguez, Gabriel de los Santos, Francisco Paz y Patricia Torres, por su colaboración en los viajes de campo. Al Museo Nacional de Historia Natural "Prof. Eugenio de Jesús Marcano" por el financiamiento y todo el apoyo a esta investigación. A los revisores anónimos por las pertinentes correcciones y sugerencias hechas al manuscrito.

\section{LITERATURA CITADA}

Almonte-Espinosa, H. y S. Latta. 2011. Aspectos del comportamiento de forrajeo de la cigüita del río Parkesia motacilla (Aves: Passeriformes: Parulidae) en época no reproductiva. Novitates Caribaea, 4: 100-108.

Arcos-Torres, I. 2005. Efecto del ancho de los ecosistemas riparios en la conservación de la calidad del agua y la biodiversidad en la microcuenca del río Sesesmiles, Copán, Honduras. CATIE, Turrialba (Costa Rica). (No. Thesis A675e). Consultado 10 de junio 2021. http://hdl.handle.net/11554/4888.

Arcos-Torres, I., F. Jiménez, C. Harvey y F. Casanoves. 2008. Riqueza y abundancia de aves en bosques ribereños de diferentes anchos en la microcuenca del río Sesesmiles, Copán, Honduras. Revista de Biología Tropical, 56 (1): 355-369.

Arendt, W. J., M. Tórrez y S. Vílchez. 2012. Diversidad de aves en agropaisajes en la región norte de Nicaragua. Ornitología Neotropical, 23: 113-131.

Chace, J. F. y J. J. Walsh. 2004. Urban effects on native avifauna: a review. Landscape and Urban Planning, 74: 46-79.

Chesser, R. T., S. M. Billerman, K. J. Burns, C. Cicero, J. L. Dunn, A. W. Kratter, I. J. Lovette, N. A. Mason, P. C. Rasmussen, J. V. Remsen, Jr., D. F. Stotz y K. Winker. 2020. Checklist of North American Birds (online). American Ornithological Society. Consultado el 10 de junio 2021. http://checklist.aou.org/taxa. 
Ding, Z., K. J. Feeley, H. Hu y P. Ding. 2015. Bird guild loss and its determinants on subtropical land-bridge islands, China. Avian Research, 6 (1): 1-9.

González-Salazar, C., E. Martínez-Meyer y G. López-Santiago. 2014. Clasificación jerárquica de gremios tróficos para aves y mamíferos de Norteamérica. Revista mexicana de biodiversidad, 85 (3): 931-941.

Gómez, M. D., L. O. Rivera, N. Politi y R. A. Ruggera. 2016. Avifauna de los bosques ribereños de las selvas pedemontanas del noroeste argentino. Ornitología Neotropical, 27: 47-57.

Guichón, M. L. y M. H. Cassini. 2007. Riparian wildlife richness along the Luján River. Ecología Austral, 17: 81-87.

Hammer, Ø., D. A. T. Harper y P. D. Ryan. 2001. PAST: Paleontological Statistics Software Package for Education and Data Analysis. Palaeontologia Electrónica, 4 (1): 9 pp.

Joseph, L. 2020. Stolid Flycatcher (Myiarchus stolidus), version 1.0. In: Birds of the World (J. del Hoyo, A. Elliott, J. Sargatal, D. A. Christie, and E. de Juana, Editors). Cornell Lab of Ornithology, Ithaca, NY, USA. https://doi.org/10.2173/bow.stofly 1.01

Kauffman, J. B., y W. C. Krueger.1984. Livestock impacts on riparian ecosystems and streamside management implications a review. Journal of Range Management, 37 (5): 430-438.

Latta, S., C. Rimmer, A. Keith, J. Wiley, H. Raffaele, K. Mcfarland y E. Fernández. 2006. Aves de la República Dominicana y Haití. Princeton University Press. VII+258 pp.

Latta, S. y K. Wallace. 2012. Ruta Barrancoli. National Aviary, 241 pp.

Lock, P. A. y R. J. Naiman.1998. Effects of stream size on bird community structure in coastal temperate forests of the Pacific Northwest, USA. Journal of Biogeography, 25 (4): 773-782.

López-Barrera, F. 2004. Estructura y función en bordes y bosques. Ecosistemas, 13: 67-77.

Marateo, G. y M. Arturi. 2013. Dinámica estacional y variación local de gremios tróficos de aves de una selva en galería y un palmar subtropical de sudamérica. Ornitologia Neotropical, 24: $213-223$.

Mastrangelo, M. E. y M. C. Gavin. 2012. Trade-offs between cattle production and bird conservation in an agricultural frontier of the Gran Chaco of Argentina. Conservation Biology, 26 (6): 1040-1051.

Mattsson, B. y R. Cooper J. 2006. Louisiana waterthrushes (Seiurus motacilla) and Habitat Assessments as Cost-effective Indicators of Instream Biotic Integrity. Freshwater Biology, 51 (10): 1941-1958.

Moreno, C. E. 2001. Método para medir la biodiversidad. M\&T-Manuales y Tesis SEA, vol. 1. Zaragoza, $84 \mathrm{pp}$.

McCrimmon Jr., D. A., J. C. Ogden, G. T. Bancroft, A. Martínez-Vilalta, A. Motis, G. M. Kirwan y P. F. D. Boesman. 2020. Great Egret (Ardea alba), version 1.0. In: Birds of the World (S. M. Billerman, Editor). Cornell Lab of Ornithology, Ithaca, NY, USA. https://doi.org/10.2173/bow.greegr.01. 
Nores, M., M. M. Cerana y D. A. Serra. 2005. Dispersal of forest birds and trees along the Uruguay River in southern South America. Diversity and distributions, 11 (3): 205-217.

Núñez, L. y E. Infante-Rivero. 2019. Composición y abundancia de la avifauna de un bosque seco tropical intervenido del Municipio Mara, Estado Zulia, Venezuela. Boletín del Centro de Investigaciones Biológicas, 53 (1): 15-37.

Palmer, G. C. y A. F. Bennett. 2006. Riparian zones provide for distinct bird assemblages in forest mosaics of south-east Australia. Biological Conservation, 130: 447-457.

Peña-Becerril, J. C., A. Monroy-Ata, F. J. Álvarez-Sánchez y M. S. Orozco-Almanza. 2005. Uso del efecto de borde de la vegetación para la restauración ecológica del bosque tropical. Tip Revista Especializada en Ciencias Químico-Biológicas, 8 (2): 91-98.

Pettingill, O. S. Jr. 1969. Ornithology in laboratory and field. Burgess, Minneapolis, Minnesota.

Ralph, C. J., S. Droege y J. R. Sauer. 1995. Managing and Monitoring Birds Using Point Counts: Standards and Applications, 161-168 pp. En: C. J. Ralph, J. R. Sauer, y S. Droege, Eds. Monitoring Bird Populations by Point Counts, USDA Forest Service, Pacific Southwest Research Station, General Technical Report PSW-GTR-149.

Ralph, C. J., G. R. Geupel, P. Pyle, T. E. Martín, D. F. DeSante y B. Milá. 1996. Manual de métodos de campo para el monitoreo de aves terrestres. Gen. Tech. Rep. PSW-GTR-159. Albany, CA: Pacific Southwest Research Station, Forest Services, U. S. Department of Agriculture, $46 \mathrm{pp}$.

Reales, C. F., G. Urich, N. Deshayes, J. J. Medrano, V. G. Alessio, E. J. León y M. A. Quiroga. 2009. Contribución al conocimiento de los gremios tróficos en un ensamble de aves de cultivo del Paraná medio. Revista FAVE Sección Ciencias Veterinarias, 8 (1): 57-65.

Ribeiro, M. D. y T. A. Melo. 2012. Dinâmica Populacional de Garças-Vaqueiras (Bubulcus ibis; Ciconiiformes: Ardeidae) no Nordeste do Estado de São Paulo. Revista Biociências, 17 (2): 25-32.

Rosetti, M. A. y A. R. Giraudo. 2003. Comunidades de aves de bosques fluviales habitados y no habitados por el hombre en el río Paraná medio, Argentina. El Hornero, 18: 89-96.

Salas-Correa, A. D. y N. Mancera-Rodríguez. 2018. Relaciones entre la diversidad de aves y la estructura de vegetación en cuatro etapas sucesionales de bosque secundario, Antioquia, Colombia. Revistan U.D.C.A Actualidad \& Divulgación Científica, 21 (2): 519-529.

Salgado-Ortiz, J. 1999. Avifauna terrestre del estado de Campeche: riqueza, abundancia y distribución de especies en los principales biomas del estado. Universidad Autónoma de Campeche. Programa de Ecología Aplicada y Manejo de Ambientes Terrestres ecomat. Informe final SNIB-CONABIO proyecto No. H324. México, DF. http:/www.conabio.gob.mx/institucion/proyectos/resultados/InfH324.pdf

Shahabuddin, G. y R. Kumar. 2006. Influence of anthropogenic disturbance on bird communities in a tropical dry forest: role of vegetation structure. Animal conservation, 9 (4): 404-413. 
Skagen, S. K., C. P. Melcher, W. H. Howe y F. L. Knopf. 1998. Comparative use of riparian corridors and oases by migrating birds in southeast Arizona. Conservation Biology, 12 (4): 896-909.

Torres-Pineda, P., C. M. Rodríguez-Peña, O. Lasso-Alcalá y A. Marmolejo. 2019. Estructura poblacional del pez endémico Limia zonata (Cyprinodontiformes: Poeciliinae) en el río Maimón, República Dominicana. Novitates Caribaea, 13: 22-38. https://doi.org/10.33800/nc.v0i13.190

Cómo citar: Almonte-Espinosa, H. (2022). Estructura y composición de un ensamblaje de aves asociadas al río Maimón, República Dominicana. Novitates Caribaea, (19), 24-42. https://doi.org/10.33800/nc.vi19.288. Artículo científico original. 